\title{
SMALL TALKS COMO ESTRATÉGIAS PARA CONVERSAÇÃO TUTORIAL EM UM ASSISTENTE DE CONHECIMENTO
}

\author{
Luciano Frontino de Medeiros \\ Grupo de Pesquisa em Educação e Novas Tecnologias (GPENT) \\ Centro Universitário Internacional UNINTER \\ luciano.me@grupouninter.com.br \\ Alvino Moser \\ Grupo de Pesquisa em Educação e Novas Tecnologias (GPENT) \\ Centro Universitário Internacional UNINTER \\ alvino.m@grupouninter.com.br \\ Neri dos Santos \\ Grupo de Pesquisa em Educação e Novas Tecnologias (GPENT) \\ Centro Universitário Internacional UNINTER \\ neri.s@grupouninter.com.br
}

\section{RESUMO}

Este artigo descreve um agente pedagógico para conversação tutorial em processos de ensino e aprendizagem a distância, com capacidade de enunciar small talks (pequenas falas) para melhorar a interatividade. A conversação tutorial que se mantém como linha mestra na comunicação com o aprendiz refere-se à apresentação de conceitos sobre o conteúdo a ser estudado de maneira formal. As estruturas de small talks são inseridas na conversação tutorial pelo agente, concebido como um Assistente de Conhecimento, para suavizar a comunicação e tornar mais informal, conferindo ainda um comportamento mais "preocupado" por parte do agente. São sugeridos 12 (doze) segmentos de small talks em fases tais como abertura e fechamento da conversação, manutenção de ritmo e controle para a gestão do aprendizado.

PALAVRAS-CHAVE: assistentes de conhecimento, conversação tutorial, small talks, agentes pedagógicos, tutoriais inteligentes.

\begin{abstract}
This paper presents a pedagogical agent for tutorial talk in learning at distance processes, capable to formulate and enunciate small talks for enhancing the interactivity. The tutorial talk that stands as a guideline in the communication refers to the concepts showing of the content which have to be studied of a formal manner. The small talks structures are inserted in the tutorial talk by the agent, designed as a knowledge assistant, to smooth the communication and become more informal, even grant a "behavior of concern" by the agent side. It's suggested twelve small talks segments in phases as talk opening and closing, rhythm maintaining and management learning control.
\end{abstract}

KEYWORDS: pedagogical agents, small talks, knowledge assistant, tutorial talk, intelligent tutorials.

\section{Introdução}

A modalidade de educação a distância tem se expandido consideravelmente ao longo dos últimos anos, em boa parte devido aos avanços tecnológicos que têm permitido o desenvolvimento de soluções inovadoras para as diferentes demandas requeridas para os processos de ensino e aprendizagem. Outro fator preponderante é o desenvolvimento da computação em nuvem, permitindo o acesso ubíquo a conteúdos massivos 
remotamente. A exigência da autonomia do aluno nesta modalidade é alta, e a falta de interação presencial necessita ser compensada pela adoção de ferramentas tecnológicas que proporcionem a mediação a distância, tais como vídeos, podcasts, chats ou ainda fóruns de discussão.

Em vista disso, é necessário aliar a noção de interatividade com as possibilidades oferecidas pela Inteligência Artificial e suas técnicas utilizando uma interface de linguagem natural para consultas aos repositórios de conhecimento feitas pelo aprendiz. Da perspectiva da interação humano-computador, Oliveira Netto (2010) afirma que sistemas de consulta a informações e sistemas baseados em conhecimento são bons exemplos para o emprego de linguagem natural. Em 1999, Liane Tarouco apresentava como perspectiva para o futuro da educação o uso de robôs de conversação nos ambientes educativos (PRIMO, 2000).

Entretanto, constata-se em que grande parte dos materiais produzidos para modalidades de ensino a distância tais como e-learning ou b-learning, apesar da riqueza de conteúdo e qualidade visual, apresentam uma característica estática, sequencial ou dependente de trilhas ou rotas predefinidas para que o aluno faça o seu estudo. A filosofia de objetos de aprendizagem pode auxiliar a diminuir esta rigidez de conteúdo, permitindo que o conhecimento seja dividido em pequenas partes autocontidas e assim maior flexibilidade tanto na concepção quanto ao uso de ambientes virtuais com objetos de aprendizagem (MEDEIROS e MUNHOZ, 2012).

A concepção de um agente pedagógico para fornecer conhecimentos mediante uma interface com linguagem natural visa oferecer ao aluno uma alternativa de estudo que se estenda além da linearidade característica dos materiais didáticos, atendendo à necessidade de aprendizagem do aluno de forma mais sistêmica e interagindo com o aluno por meio de linguagem natural. Os objetos e conceitos referentes a um tema específico podem ser recuperados conforme perguntas efetuadas pelo aluno ao sistema, de acordo com uma estrutura que possibilite a busca aproximada de termos, em uma estratégia de aprendizagem que vá mais além da puramente behaviorista (MEDEIROS e MARTINS, 2012).

Seguindo esta hipótese, o modelo de comunicação envolvido na conversação com o aluno deve se estender além da conversação tutorial propriamente dita e ser permeado pelo uso de small talks ${ }^{1}$. Estudadas na área da linguística, small talks são pequenas falas que tem a função de manter o canal de comunicação aberto entre os interlocutores, facilitando a interatividade através de falas e temas que são comuns em uma conversação humana (JAKOBSEN, 1960 apud BICKMORE, 1999). São utilizadas, por exemplo, para abrir ou fechar uma conversa, ou lançar outros temas não relacionados ao contexto da conversa e ainda evitar silêncios estranhos. Técnicas baseadas em small talks teriam a função de facilitar a interatividade e auxiliar na promoção da aprendizagem por parte do aluno, com a possibilidade de proporcionar um contexto emocional positivo para o processo de ensino e aprendizagem.

Assim, este artigo apresenta um agente pedagógico denominado de "Assistente de Conhecimento" (AC) cujo modelo de comunicação é baseado na conversação tutorial propriamente dita e na conversação por small talks. O texto a seguir aprofunda-se na teoria de small talks, iniciada nos estudos de Malinowski, abordando também os robôs de conversação e os chatterbots educacionais. Apresenta-se ainda uma breve descrição

\footnotetext{
${ }^{1}$ De acordo com o Dicionário Michaelis, o termo composto small talk é traduzido para o português como "conversa fiada" ou "tagarelice". Preferiu-se manter aqui a denominação original em inglês para não haver equívocos de entendimento quanto à importância para a linguagem e ao propósito deste artigo.
} 
da estrutura do AC e seu modelo de comunicação contendo estratégias de uso de small talks. Discute-se por fim alguns pontos de interesse e fatores críticos sobre esta abordagem, concluindo-se com as considerações finais.

\section{Small Talks}

De acordo com Bickmore (1999), existem dois elementos chave que permitem a um software estabelecer credibilidade em uma interação com um ser humano. A primeira é dar um "corpo" ao agente numa interface, ou seja, fornecer uma aparência e humana junto com um comportamento. Um segundo mecanismo é mediante o uso de small talks. A efetividade do uso de small talks sobre a percepção do usuário de um agente de software tem sido demonstrada em sistemas que implementam chatterbots, de forma semelhante ao ELIZA de Weizenbaum (1976).

Small talks são conversações, sem orientação à tarefa, que versam sobre tópicos neutros cujas funções primárias são mitigar a ameaça facial, fornecer um intervalo inicial no qual os interlocutores podem comparar-se um com o outro, estabelecer um estilo interacional e firmar algum grau de confiança mútua e relacionamento (BICKMORE e CASSELL, 1999). Os encontros sociais entre indivíduos que nunca tiveram algum encontro ou não são familiares entre si são usualmente iniciados por pequenas falas nas quais uma conversação leve é feita sobre tópicos de interesse geral (BICKMORE, 1999).

O estudo de small talks na área da linguística começa com o trabalho seminal de Malinowski em 1926, o qual definiu o termo "comunhão fática” como um tipo de fala na qual laços de união são criados mediante uma mera troca de palavras. As small talks seriam uma espécie de linguagem utilizada no relacionamento cotidiano, ocorrendo quando as pessoas estão mais à vontade ou quando acompanham algum trabalho manual por conversas desconectadas do que estão fazendo (BICKMORE, 1999). Uma das razões essenciais para utilizar small talks é evitar o silêncio que se origina de uma tensão desconfortável que pode ter consequências negativas para a interação social. Mesmo a taciturnidade é considerada em algumas culturas como um sinal evidente de hostilidade ou mau humor (CRUZ, 2005).

Jakobsen (1960) descreve as small talks possuindo uma função “fática”, cujo objetivo é o de manter um canal aberto de comunicação entre os interlocutores, além do conteúdo da mensagem, do emissor e do receptor.

Schneider (1988) apresentou um estudo bastante extenso sobre diversos gêneros de small talks, oferecendo uma perspectiva com duas funções básicas: uma assinalando as necessidades de "face positiva”, preenchendo uma função social; e outra atribuindo necessidades de "face negativa", evitar situações de embaraço ou o silêncio perigoso com um estranho. Schneider transcreveu cerca de 30 tipos de conversações e fez uma detalhada caracterização de small talks. Sua abordagem ressalta as small talks acontecendo tipicamente em séries de segmentos conversacionais, acontecendo como uma sequência de movimentos.

Coupland et al (1992), por outro lado, oferecem uma perspectiva diferenciada na qual as small talks não acontecem em frames discretos de interação, mas indo além em um estilo de interação que preenche a função de facticidade de forma continua, apresentando níveis variados de facticidade baseados nas necessidades momentâneas dos interlocutores para gerenciar a sua distância interpessoal.

Bickmore e Cassell (1999) apresentam um agente para venda de imóveis que contém uma interface visual e um modelo conversacional baseado em narrativas e small talks. 
Justificam a sua proposta na asserção de que um agente que se pareça com um ser humano torna mais fácil a construção de confiança. A possibilidade de contar histórias e efetivar small talks por parte do agente requer o planejamento cuidadoso dos discursos que podem ser enunciados.

\section{Robôs de Conversação}

Os chatterbots ou robôs de conversação podem ser considerados como a realização prática da proposta de simulação de inteligência lançada por Alan Turing em 1950 no seu artigo seminal “Can machines think?”. De acordo com sua previsão, por volta do ano 2000 a capacidade de processamento dos computadores atingiria a escala de $10^{9}$ bits, estando aptos a emular o comportamento verbal e confundir um interlocutor quanto à sua procedência "biológica ou tecnológica” (MAULDIN, 1994).

Relacionado ainda a sistemas como agentes com capacidade de comunicação em linguagem natural, o software ELIZA de Joseph Weizenbaum é um dos mais emblemáticos, o qual simulava a conversa de um psicólogo rogeriano no atendimento a um paciente humano. Mesmo o ELIZA tendo status de referência para os sistemas tutoriais inteligentes, Weizenbaum adotou uma postura bastante pessimista quanto à possibilidade de sistemas com interface de linguagem natural que não possuíssem domínios de conhecimento ou oferecessem soluções para problemas realmente relevantes (WEIZENBAUM, 1976).

A efetividade da interação do usuário com um robô de conversação está relacionada diretamente à capacidade do usuário em perceber uma "mente”, um grau de inteligência com credibilidade suficiente, na outra ponta da conversação. Isto vai ao encontro da ideia de sistema intencional de Daniel Dennett. A constatação de uma postura intencional é a estratégia de interpretar o comportamento de uma entidade qualquer, tratando-a como se fosse um agente com racionalidade, com condições de governar suas escolhas de ação por uma consideração de suas crenças e desejos. A postura intencional é a atitude ou perspectiva que é rotineiramente adotada na relação com o outro. Portanto, adotar a postura intencional em relação a alguma "outra coisa” parece ser a antropomorfização desta "outra coisa”. A estratégia básica da postura intencional é tratar a entidade em questão como um agente, para predizer e em certo sentido explicar suas ações e seus movimentos (DENNETT, 2006).

\section{Chatterbots Educacionais}

A existência da competição Loebner, que premia anualmente desde 1991 os sistemas próximos de atingirem o ideal de Turing, é um incentivo ao desenvolvimento de sistemas cada vez mais complexos e capazes de iludir uma comissão julgadora. Se tais sistemas conversacionais são feitos para emularem a conversação com seres humanos, apresentando resultados satisfatórios, por que não considerar seu potencial uso em conversação tutorial, dentro de processos de ensino e aprendizagem EAD?

Várias propostas foram feitas quanto ao desenvolvimento de chatterbots educacionais. Primo et al (2000), propõe o uso de chatterbots para a educação a distância, relatando a percepção de alunos do ensino fundamental com dois chatterbots experimentais. Podese destacar também o ELEKTRA, um robô de conversação para o ensino de física e redes de computadores, baseado no ALICE, um chatterbot com código open source bastante conhecido (LEONHARDT et al, 2003).

Chatterbots têm sua funcionalidade baseada em análises de perguntas enunciadas pelo aluno e buscas de contexto, tendo um comportamento reativo. Sistemas que pudessem captar as reações do aluno além do texto em si poderiam ser mais eficazes. Apresentando um design mais complexo e contendo um framework para inferência de 
reações emocionais do aluno e também de suporte emocional, agentes pedagógicos para interatividade afetiva com alunos têm sido propostos tais como o PAT (JACQUES e VICARI, 2003).

Castanho e Wazlawick (2002) relatam o desenvolvimento de uma ferramenta de autoria para facilitar a tarefa de criação de chatterbots por usuários, mediante o uso de linguagem natural. Andrade (2012) propõe o desenvolvimento de um chatterbot educacional para uso em dispositivos móveis com domínio de conhecimento sobre a internet.

O uso de chatterbots educacionais pode trazer uma série de vantagens (PRIMO et al, 2000):

- Oferecer um tipo de interação mais humanizada, ficando na dependência do seu potencial comunicativo;

- Podem ser excelentes fontes de reforço de conteúdo, fontes de pesquisa ou mesmo auxiliando as tarefas dos professores ou ainda oferecendo links para pesquisas.

Outras vantagens podem ser adicionadas a estas (CASTANHO e WAZLAWICK, 2002):

- Disponibilidade ininterrupta do chatterbot, trazendo inúmeras vantagens no caso do ensino a distância;

- Utilização como ferramenta de estudo tanto em sala de aula como fora dela, devido à sua automatização.

\section{O Assistente de Conhecimento}

O AC é um agente pedagógico que tem o objetivo de proporcionar os conhecimentos armazenados sobre um domínio específico, estruturado na forma de ontologias, tendo uma interface de linguagem natural adaptativa com relação às questões formuladas pelos alunos. Considera-se, portanto, uma estrutura de conversação tutorial onde o AC fornece respostas às consultas efetuadas pelo usuário/aprendiz. Este nível de interação tutorial presume o uso de uma estratégia instrucionista, onde prevaleceria a transmissão de conteúdos de forma síncrona ao aluno.

A modelagem de um chatterbot educacional deve levar em consideração os objetivos educacionais pretendidos com relação ao contexto de ensino e aprendizagem em questão. Justifica-se o nome "assistente de conhecimento" pela definição de seus objetivos cognitivos, fundamentando-se na taxonomia de Bloom. Aqui, os dois primeiros níveis de aprendizagem são o nível de conhecimento e o nível de compreensão. No nível de conhecimento, incluem-se os comportamentos que dão ênfase quanto à recordar unidades de informação suscetíveis de serem assimiladas. No nível de compreensão, existe a expectativa de que os alunos entendam o que se transmite e que possam fazer uso das ideias que possuem (PERES e PIMENTA, 2011).

O AC contém dois módulos principais: uma base de conhecimento (BC) e o módulo de interface de linguagem natural (ILN), composto por sua vez de uma série de agentes com tarefas específicas. No módulo BC, o agente de buscas diretas e o agente de buscas probabilísticas são responsáveis por efetuá-las em uma série de ontologias de domínio, referentes aos conteúdos dos cursos em questão. Estas ontologias permitem ao AC fornecer, através do módulo ILN no qual o aluno insere suas perguntas, as respostas de acordo com representações ontológicas objeto-atributo-valor (cláusulas OAV). Tais respostas são fornecidas pelos agentes de respostas diretas e o agente de conversação (MEDEIROS e MARTINS, 2012). 
Dentro do módulo ILN, o AC possui uma base inicial de perguntas e respostas, manipulada pelo agente de respostas diretas. Quando a pergunta não é solucionada pelo agente de resposta direta, o agente de busca probabilística processa as questões dos alunos através de comparações utilizando quatro (4) diferentes métricas para aferição de proximidade sintática, considerando tanto palavras individuais quanto compostas como objetos de aprendizagem. Os agentes de buscas, tanto direta quanto probabilística, ao contrário da maioria dos sistemas de linguagem natural, não procedem à análise sintática conforme a gramática da linguagem, atendo-se aos requisitos do conhecimento armazenado na forma de cláusulas OAV e a um processo de busca direta ou por proximidade baseado nelas.

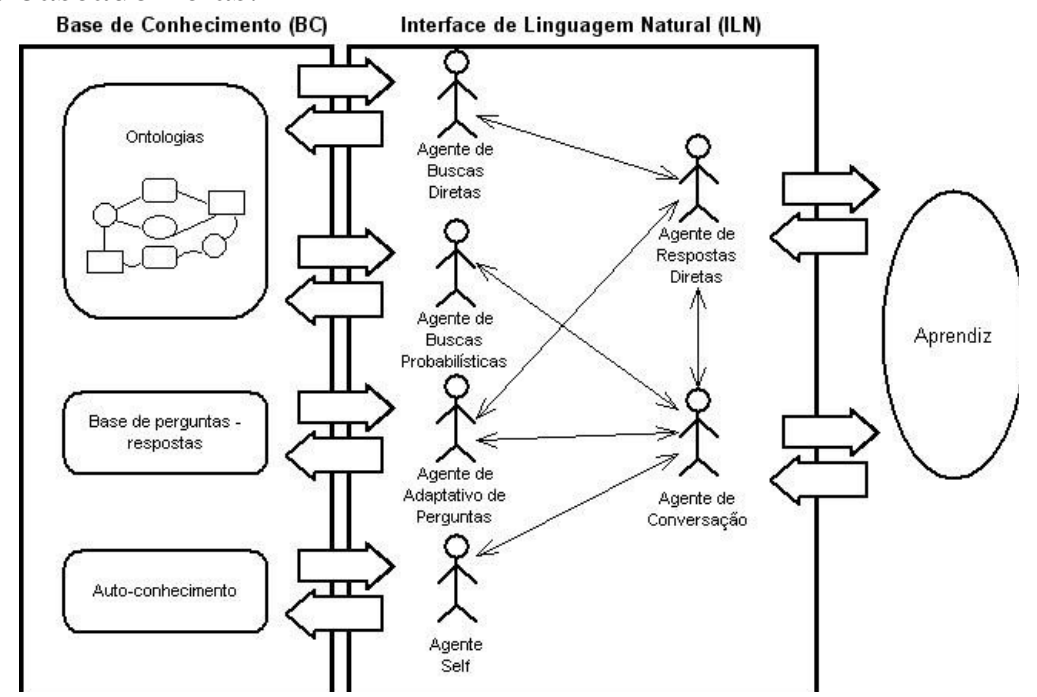

Figura 1 - Diagrama esquemático do Assistente de Conhecimento, mostrando as interações entre os agentes.

Caso as perguntas feitas pelos alunos-usuários não façam parte da base de perguntasrespostas, estas serão armazenadas, considerando-se uma classificação coerente com as métricas de proximidade sintática, através do agente adaptativo. Por meio do feedback ou da confirmação dada pelo aluno, o AC tem condições de se adequar ao perfil individual ou coletivo de interação, emergindo assim um quadro de interatividade de natureza diversificada e trazendo uma característica de aprendizagem de máquina ao AC. Portanto, em função desta característica, o AC tem condições de se adaptar a diversos contextos de conversação.

Além das vantagens adquiridas dos chatterbots educacionais, o AC também proporciona outras adicionais:

- Regularidade e conformidade de conteúdo, desde que a ontologia de domínio seja construída adequadamente e sem ambiguidades;

- Divisão do conteúdo de um objeto de aprendizagem em partes ainda menores, com a granularidade em nível de conceitos, exemplos, classificações, taxonomias, etc.

\section{Small Talks e o Assistente de Conhecimento}

O conceito de small talks utilizado neste trabalho considera como não orientada à tarefa, por exclusão, toda conversação que não esteja relacionada à recuperação de conteúdos por meio de questões, por parte do aluno. Desta forma, uma grande gama de enunciações no AC pode ser contextualizada como small talks. Dentro da interface de linguagem natural do AC, o uso de small talks é distribuído em momentos diferenciados 
da conversação tutorial, sendo resumidos até o presente em 12 (doze) segmentos, de acordo com a perspectiva discreta de Schneider, descritos a seguir:

- Abertura da conversação: a saudação inicial, contextualizando ao fato do aluno acessar a primeira vez ou acessar novamente. Esta saudação não é determinada, havendo uma série de enunciações que são apresentadas de forma probabilística ao aluno;

- Fechamento da conversação: seguindo a mesma forma probabilística de enunciação da abertura;

- Autoconhecimento: refere-se ao self do AC, relacionado a um conhecimento embutido que sustenta a personalidade, concedendo-se ao AC um aspecto mais "humano" e permitindo que se apresente ao aluno, mostrando suas funcionalidades;

- Intervalos de Silêncio: quando o aluno não interage com o AC durante algum tempo, o AC tenta retomar fazendo perguntas sobre a presença do aluno, lançando perguntas para consultar novos objetos; Em caso negativo de interação, o AC presume que o aluno não se encontra mais disponível e encerra a conversação;

- Pausas no andamento: relacionado ao período em que o aluno está conectado, o $\mathrm{AC}$ enuncia ao aluno os objetos que ele já pesquisou e pergunta se quer fazer uma pausa;

- Frases retóricas: pequenas frases, colocadas de forma aleatória e diversificada antes das respostas sobre conteúdos, indicando que o AC está "pensando" antes de fornecer a resposta;

- Sugestão de conteúdo: lançado após uma pesquisa sobre que conteúdos o AC sabe sobre um objeto especificamente. De forma aleatória, o AC indica ao aluno se ele quer acessar um determinado conteúdo sobre um objeto, sujeito ao aceite do aluno;

- Gestão do aprendizado: mediante perguntas do aluno, o AC pode informar o status do que já foi estudado, em termos de objetos já visualizados, o que falta estudar, sendo apresentado inclusive de forma quantitativa;

- Contato com o tutor: para aqueles objetos que não estejam na ontologia, ou no caso de não haver resposta para a pergunta, o AC indica que contatará o tutor para enviar a pergunta que foi feita;

- Ponto de retomada: em novas conexões que o aluno venha a fazer, após conexões onde houve um intervalo de silêncio, o AC indica ao aluno, após a abertura, os últimos objetos que foram pesquisados;

- Confirmação de objeto: antes de acionar o segmento de contato com o tutor sobre uma pergunta identificada com baixa probabilidade, o AC prioriza três perguntas com maior proximidade e as apresenta ao aluno, requisitando confirmação. Em caso positivo sobre alguma delas, a pergunta original é associada a esta indicada pelo aluno, e armazenada na base de perguntasrespostas;

- Gentileza: respostas positivas a agradecimentos do aluno.

No quadro 1 é mostrado um trecho de conversação tutorial com o AC, onde é possível identificar alguns segmentos de small talks inseridas na conversação. Para manter a operacionalidade do AC em um nível que possa estabelecer continuidade de comunicação com o aluno, deve-se ter o cuidado com alguns parâmetros tais como: 
- Tempo de resposta: a resposta ao aluno não deve ser mostrada de imediato, nem demorar muito para ser apresentada;

- Aleatoriedade de resposta: a opção de resposta, tanto diretas quanto à relacionada com o conhecimento da ontologia, deve ser diversificada, na medida que dê a impressão de um vocabulário rico;

- Controle de responsividade: em certos momentos da conversação, quando determinados segmentos de small talks são apresentadas, deve haver um retorno positivo ou negativo do aluno;

- Continuidade tutorial: o AC deve manter uma continuidade quando sugere elementos ao aluno, buscando incentivar objetos que não foram estudados ou pesquisados até o momento;

- Conhecimento compartimentado: o conhecimento é granular, armazenado em pequenas partes na ontologia que podem ser recuperados por perguntas simples.

Quadro 1 - Trecho de uma conversação com o AC, mostrando alguns segmentos de small talks durante a conversação tutorial.

\begin{tabular}{|c|c|}
\hline Trecho de conversação tutorial & Segmento de small talk \\
\hline $\begin{array}{l}\text { [AC]: Oi, <Usuário>! Como vai? } \\
\text { [AC]: Seja bem-vindo! Fique à vontade para perguntar. } \\
\text { [Usuário]: Obrigado } \\
\text { [AC]: À sua disposição! } \\
\text { [Usuário]: Quem é você? } \\
\text { [AC]: Sou AC, agente de conhecimento para a disciplina de SIG } \\
\text { [AC]: Tenho armazenado alguns objetos de aprendizagem desta disciplina. } \\
\text { [AC]: Pode fazer perguntas diretas sobre o conteúdo desta disciplina, assim como: } \\
\text { 'qual o conceito disto', 'existe relação entre isto e aquilo'. Ok? } \\
\text { [Usuário]: Ok } \\
\text { [Usuário]: O que é informação? } \\
\text { [AC]: conforme Davenport e Prusak, informação quer dizer uma mensagem, } \\
\text { geralmente na forma de um documento ou uma comunicação audível ou visível } \\
\text { [Usuário]: Pode me dar um exemplo? } \\
\text { [AC]: Referente a informação? } \\
\text { [Usuário]: Sim } \\
\text { [AC]: por exemplo, informação pode ser um informativo de comunicação interna } \\
\text { [AC]: Quer perguntar mais alguma coisa? } \\
\text { [Usuário]: Sim } \\
\text { [Usuário]: O que você sabe sobre informação ? } \\
\text { [AC]: Encontrei } 6 \text { conceitos, } 4 \text { exemplos, } 1 \text { comentário, } 3 \text { links, } 2 \text { classificações, } 7 \\
\text { relações e } 1 \text { sinônimo. } \\
\text { [AC]: Gostaria de ver um link? } \\
\text { [Usuário]: Sim } \\
\text { [AC]: tenho o seguinte link: Dado, informação e conhecimento: uma reflexão } \\
\text { (http://webinsider.uol.com.br/2004/10/13/dado-informacao-e-conhecimento-uma- } \\
\text { reflexao/) } \\
\text { [AC]: Quer abrir o link? } \\
\text { [Usuário]: Sim } \\
\text { [AC]: O site não é válido! } \\
\text { [AC]: Vou enviar isto ao tutor para que ele verifique! }\end{array}$ & $\begin{array}{l}\text { Confirmação de objeto } \\
\text { Intervalo de silêncio } \\
\text { Gestão do aprendizado } \\
\text { Sugestão de conteúdo }\end{array}$ \\
\hline
\end{tabular}

\section{Discussão}

A implementação de um sistema inteligente assim como o AC para processos de conversação tutorial pode induzir ao pensamento equivocado ou a um otimismo exacerbado que implique, de forma prematura, na ideia da substituição de tutores humanos. É importante então ressaltar as seguintes posições relativas ao papel a ser desempenhado pelo AC:

- O objetivo é fazer com que o sistema responda a perguntas efetuadas pelo usuárioaprendiz, numa perspectiva instrucionista, ou seja, de transmissão de novos conhecimentos mediante uma interface de linguagem natural. Isto já foi salientado anteriormente, quando da definição dos objetivos cognitivos baseados na taxonomia de Bloom. Um professor ou tutor humano também possui tal competência; porém, o 
AC pode, levando-se em consideração seu determinismo relativo, ser mais eficiente neste processo de transmissão de conteúdo, até mesmo em função da própria padronização que pode agregar ao processo de tutoria;

- Devido ao AC se constituir num “sistema”, a postura por parte do aluno em relação a uma "coisa" que está lhe transmitindo novos conhecimentos pode recair em uma subestimação, ou mesmo em uma atitude de desdém com o AC, quanto ao seu desempenho efetivo dentro do processo de ensino e aprendizagem. Em vista disto, a consideração do AC como um sistema intencional se faz necessária; o AC deve parecer "inteligente" o suficiente para fazer com que o aluno adote a postura intencional ao invés de uma simples postura de projeto. Ainda que na fase de desenvolvimento tenha se predito uma série de situações de interação, versões mais robustas precisam contemplar o feedback proveniente de diferentes alunos;

- O uso de small talks tende a auxiliar nesta adoção da postura intencional por parte do aluno, em facilitar a comunicação e manter o canal aberto com o aluno, provendo uma dinâmica de conversação além da simplesmente formal. Mas o uso de small talks não deve extrapolar o âmbito da conversação tutorial, ou ser utilizada em demasia, sob pena de "artificializar" a conversação ou mesmo ser inoportuna quanto ao objetivo principal que é fornecer conhecimentos ao aluno.

Desta forma, a integração de small talks em uma conversação oferece uma série de desafios e também dificuldades. Bickmore (1999) assinalou algumas delas, adaptadas a seguir:

- Caracterização de estados contínuos de small talks, a facticidade, podendo ocorrer em diferentes níveis além dos segmentos discretos relatados por Schneider (1980);

- Melhor avaliação da proximidade interpessoal, o que pode requerer métodos plausíveis de estimação psicológica ou appraisal, tais como os utilizados em computação afetiva (JACQUES e VICARI, 2005).

- Modos diversos de small talks, ou seja, quando a conversação foge da orientação à tarefa, incluído aí a dinâmica pelo surgimento de novas formas de small talks;

\section{Considerações Finais}

Na fase atual de desenvolvimento do protótipo, o AC contém uma ontologia parcial do domínio de conhecimento referente à área de sistemas de informações gerenciais. Aqui, já é evidenciado o potencial de utilização prática ao longo das simulações de interação conversacional. Ao final da construção desta ontologia, o AC será testado com um grupo de controle em um curso específico por um dos autores, em um ambiente virtual de aprendizagem.

É válido ressaltar que a adoção de um AC em um processo de ensino e aprendizagem não visa à substituição completa de um professor ou tutor, junto ao aprendiz. Um AC representará uma ferramenta inteligente a ser adicionada ao conjunto de meios, que devem ser disponibilizados aos aprendizes para melhorar o aprendizado de conceitos. $\mathrm{O}$ processo de tutoria presume a mediação de um professor ou tutor, no sentido da manutenção e evolução do conhecimento armazenado em um AC, tornando-o um repositório de conhecimento, tal como um livro ou e-book, porém com uma característica maior de interatividade diferenciada e perspectivas de adaptabilidade ao aluno. Neste contexto, se os agentes são capazes de enunciar small talks durante uma conversação tutorial, crê-se que as chances de sucesso com relação à aprendizagem possam ser sensivelmente aumentadas. 


\section{Referências}

ANDRADE, R. M. Mobile bot: um chatterbot educacional para dispositivos móveis. Revista Brasileira de Computação Aplicada, Passo Fundo, v.4, n.2, p.83-91, out-2012.

BICKMORE, T. A computational model of small talk. MAS 962 Discourse and Dialogue for Interactive Systems, 1999. http://web.media.mit.edu/ bickmore/Mas962b/. Acesso em 10/04/2013.

BICKMORE, T.; CASSELL, J., Small Talk and Conversational Storytelling. In: Embodied Interface Agents in Proceedings of the AAAI Fall Symposium on Narrative Intelligence, 1999. Disponível em http://www.media.mit.edu/gnl/ publications.html. Acesso em 20/04/2013.

COUPLAND, J.; COUPLAND, N.; ROBINSON, J. D. How are you? Negotiating phatic communion. Language in Society n.21, p.207-230, 1992.

CRUZ, M. P. On the Phatic Interpretation of Utterances: A Complementary Relevance-Theoretical Proposal.

DENNETT, D. C. Brainstorms: escritos filosóficos sobre a mente e a psicologia. São Paulo: Ed. UNESP, 2006.

FLÔRES, M. L. P.; VICARI, R. M. Inteligência Artificial e o Ensino com Computador. RENOTE-Revista de Novas Tecnologias na Educação, v.3, n.1, maio/2005. Disponível em http://seer.ufrgs.br/renote/article/view/13938/7841. Acesso em 10/04/2013.

JAQUES, P. A.; VICARI, R. M. PAT: Um Agente Pedagógico Animado para Interagir Afetivamente com o Aluno. RENOTE-Revista de Novas Tecnologias na Educação, $\quad$ v.3, n.1, maio/2005. Disponível em http://seer.ufrgs.br/renote/article/view/13738/7967. Acesso em 10/04/2013. LEONHARDT, M. D.; CASTRO, D, D.; DUTRA, R. L. de S.; TAROUCO, L. M. R. ELEKTRA: Um Chatterbot para Uso em Ambiente Educacional. RENOTE-Revista de Novas Tecnologias na Educação, v.1, n.2, setembro/2003. Disponível em http://seer.ufrgs.br/renote/article/view/14336/8251. Acesso em 10/04/2013.

MAUldin, M. L. Chatterbots, Tinymuds, and the Turing Test Entering the Loebner Prize Competition. Proceedings of AAAI, 1994. Disponível em http://aaaipress.org/Papers/AAAI/1994/AAAI94-003.pdf. Acesso em 20/04/2013. MEDEIROS, L. F.; MARTINS, O. B. Knowledge Assistant with Natural Language Interface and Multiagent Architeture for Mentoring Process in Education at Distance. $9^{\circ}$ CONTECSI- Congresso Internacional de Tecnologia e Sistemas de Informação. São Paulo, FEA-USP, 2012.

OLIVEIRA NETTO, A. A. IHC e a Engenharia Pedagógica: interação humano computador. Florianópolis: Visualbooks, 2010.

PERES, P.; PIMENTA, P. Teorias e Práticas de B-Learning. Lisboa: Sílabo, 2011. PRIMO, A. F. T.; COELHO, L. R.; PAIM, M. F. R.; REICHEL, D. O uso de chatterbots na educação a distância, 2000. Disponível em http://www.nied.unicamp.be/oea. Acesso em 25/04/2013.

RODRIGUES, L. M. L.; CARVALHO, M. STI-I: Sistemas Tutoriais Inteligentes que integram Cognição, Emoção e Motivação. Revista Brasileira de Informática na Educação, v.13, n.1, p.20-34, 2005. Disponível em http://www.brie.org/pub/index.php/rbie/article/view/55/61. Acesso em 13/04/2013.

WEIZENBAUM, J. O Poder do Computador e a Razão Humana. Lisboa: Edições 70, 1976. 